Artículo Original. Mayo-Agosto 2017; 7(2):43-59. Recibido: 24/01/2017 Aceptado: 03/04/2017.

http://dx.doi.org/10.21929/abavet2017.72.4

\title{
Efecto del consumo de zinc orgánico en la respuesta productiva de la cerda y su camada
}

Effect of organic zinc intake on the productive response of sows and their litter

\section{Romo-Valdez Juan1 romo_14@hotmail.com Romo-Rubio Javier¹ romo60@uas.edu.mx Barajas-Cruz Rubén'1 rubar@uas.edu.mx Enríquez-Verdugo Idalia1 idaliaenver@yahoo.com.mx Gabriela Silva-Hidalgo'1 gabsilhid@uas.edu.mx Montero-Pardo Arnulfo' arnulfomp@hotmail.com}

${ }^{1}$ Facultad de Medicina Veterinaria y Zootecnia de la Universidad Autónoma de Sinaloa. Culiacán, México. *Autor responsable y de correspondencia: Romo-Rubio Javier. Facultad de Medicina Veterinaria y Zootecnia de la Universidad Autónoma de Sinaloa; Boulevard San Ángel s/n, Colonia San Benito, Culiacán, Sinaloa, México, CP 80246.

\section{RESUMEN}

Para evaluar la respuesta productiva de la cerda y su camada a la suplementación con zinc orgánico en clima tropical, se realizaron dos experimentos. Exp. 1 (Época fresca). Se utilizaron 46 cerdas Yorkshire $x$ Landrace, asignadas a uno de dos tratamientos (T) en un diseño completamente al azar. T1 ( $S Z n ; n=22)$; dieta sin adición de $Z n$ a partir de los 35 días de gestación y durante 21 d de lactación; T2 $(C Z n ; n=24)$ T1 más la adición de $100 \mathrm{mg}$ de Zn/kg de alimento. Exp. 2 (Época cálida). Se utilizaron 44 cerdas, asignadas al azar a uno de dos T similares al Exp.1: T1 $(S Z n ; n=25)$ y T2 $(C Z n ; n=19)$. Los resultados fueron analizados por ANDEVA $(P \leq 0.05)$. Resultados: Exp. 1. El consumo de alimento adicionado con $\mathrm{Zn}$ incrementó $(P=0.001)$ la concentración plasmática de lgG en los cerdos destetados. Exp. 2 . El consumo de alimento adicionado con $\mathrm{Zn}$, incrementó $(P<0.05)$ el espesor de grasa dorsal $(E G D)$ de las cerdas durante la gestación (16.6 vs. $14.8 \mathrm{~mm}$ ) y disminuyó $(\mathrm{P}=0.006)$ la mortalidad durante la lactancia (11 vs. $26 \%$ ). Se concluye que el consumo adicional de $\mathrm{Zn}$ incrementa el EGD en las cerdas gestantes bajo condiciones de estrés calórico y disminuye la mortalidad de lechones durante la lactancia, y el consumo adicional durante la época fresca incrementa los niveles plasmáticos de lgG en los LD.

Palabras clave: Metionina de Zinc, Espesor de grasa dorsal, Mortalidad predestete.

\begin{abstract}
To evaluate the productive response of sows and their litter to the supplementation with zinc in a tropical climate two experiments were realized. Exp. 1 (Fresh season). 46 Yorkshire $x$ Landrace sows were used, assigned to one of two treatments $(T)$ in a completely randomized design. T1 $(S Z n ; n=22)$; diet without addition of $Z n$ from 35 days of gestation and during $21 \mathrm{~d}$ of lactation, and T2 (CZn; $n=24)$, T1 diet plus supplementation with $100 \mathrm{mg} \mathrm{Zn/kg}$ of feed. Exp. 2 (Warm season). Another 44 sows were assigned to one of two treatments similar to Exp. 1. $T 1(S Z n ; n=25)$ and T2 $(C Z n ; n=19)$. Results were analyzed by ANOVA $(P \leq 0.05)$. Results: Exp. 1. Feed consumption added with $Z n$ increased $(P<0.05)$ lg $\mathrm{Clasmatic}$ concentration in weaned pigs. Exp. 2. Feed consumption added with $\mathrm{Zn}$ increased $(P<0.05)$ backfat thickness (BFT) of the sows during the gestation (16.6 vs. $14.8 \mathrm{~mm})$ and decreased $(P=0.006)$ the mortality of nursing pigs during the lactation (11 vs. $26 \%$ ). It concludes that additional consumption of zinc increase the BFT in gestating sows under environment heat stress and diminished the mortality of nursing pigs during lactation and intake of supplemented diet with zinc during fresh season increase IgG plasmatic concentration levels in weaned pigs.
\end{abstract}

Keywords: zinc, sow, backfat thickness, nursing pig mortality. 


\section{INTRODUCCIÓN}

El zinc (Zn) es un nutriente esencial en la dieta de los cerdos (NRC, 2012; Hill et al., 2014). Es un mineral traza con demostrada importancia para la función de más de 300 enzimas (Bhowmik et al., 2010; Chasapis et al., 2012), influyendo en el equilibrio ácidobase, la competencia inmune y las funciones celulares básicas (Haase y Rink, 2009a; Kelleher et al., 2011). La acción metabólica del Zn incluye el metabolismo energético, la síntesis de proteínas, metabolismo de los ácidos nucleicos, la integridad del tejido epitelial, la reparación y la división celular, transporte y utilización de la vitamina A, y la absorción de vitamina $\mathrm{E}$ (Bhowmik et al., 2010; Borah et al., 2014).

Se ha demostrado que el Zn dietético mejora y previene la reducción de la integridad intestinal durante el estrés calórico (Sanz et al., 2014; Pearce et al., 2015), disminuye la permeabilidad intestinal de los lechones durante el destete (Zhang y Guo, 2009), promueve la restauración del epitelio intestinal (Hu et al., 2014; Song et al., 2015) y mejora el metabolismo proteico en el cerdo (Pearce et al., 2015). Debido a que los requerimientos de Zn se incrementan durante el estrés calórico (Lagana et al., 2007), se ha sugerido que la suplementación con Zn podría utilizarse para atenuar la disminución sérica del Zn durante periodos de altas temperaturas ambientales (Chand et al., 2014; Li et al., 2015).

Las dietas para cerdos son generalmente complementadas con $\mathrm{Zn}$ inorgánico ( $\mathrm{ZnSO}{ }^{4} \mathrm{u}$ $\mathrm{ZnO}$ ) para asegurar el aporte requerido, siendo la fuente inorgánica de $\mathrm{ZnSO}^{4}$ la de mayor biodisponibilidad (NRC, 2012).

En la granja donde se realizó el estudio la fuente de $\mathrm{Zn}$ utilizada es $\mathrm{ZnSO}^{4}$. Las dietas animales a menudo contienen antagonistas que reducen la biodisponibilidad de las formas inorgánicas de $\mathrm{Zn}$, creando así una deficiencia. Varios estudios sugieren que las fuentes orgánicas de $\mathrm{Zn}$ son más biodisponibles que las formas inorgánicas, y la biodisponibilidad de las formas orgánicas respecto de las inorgánicas aumenta dramáticamente en presencia de antagonistas como el $\mathrm{Ca}, \mathrm{P}$, ácido fítico y fibra cruda (Bao et al., 2007; Nollet et al., 2007; Schlegel et al., 2013; Richards et al., 2015). Además, en un estudio reciente Ming-Zhe et al. (2016), observaron que el valor biológico del zinc orgánico a partir de metionina de zinc (Met-Zn), fue de 64\% mayor que el del sulfato de zinc. Es por ello, que el objetivo del presente estudio fue evaluar la respuesta productiva de la cerda gestante y su camada, al consumo de dietas adicionadas con Zn orgánico a partir de metionina de zinc, bajo condiciones de clima tropical.

\section{MATERIAL Y MÉTODOS}

Se realizaron dos experimentos en la granja porcina "La Huerta", localizada en la Sindicatura de Culiacancito, Culiacán, Sin., con coordenadas geográficas: $24^{\circ} 49^{\prime} 38^{\prime}$ 
latitud Norte y $107^{\circ} 22^{\prime} 47^{\prime}$ longitud Oeste, con una altitud de $60 \mathrm{msnm}$. El clima se clasifica como semiseco muy cálido (BS1(h')), con temperatura media anual de $24.9^{\circ} \mathrm{C}$, con máximas de $45^{\circ} \mathrm{C}$ en los meses de julio y agosto, y mínimas de $7^{\circ} \mathrm{C}$ en diciembre y enero. La precipitación pluvial es de $671.4 \mathrm{~mm}$, con precipitaciones máximas en los meses de julio, agosto y septiembre.

\section{Experimento1.}

Diseño experimental: se utilizaron 46 cerdas multíparas híbridas (Yorkshire x Landrace), a las que se les asignó uno de dos tratamientos, en un diseño experimental completamente al azar. Los tratamientos $(T)$ consistieron en: T1 $(S Z n ; n=22)$; recibieron una dieta, a base de maíz-pasta de soya con aporte nutrimental de acuerdo a la etapa fisiológica, a partir de los 35 días de gestación (79 días, con un consumo de $2 \mathrm{~kg}$ alimento/día), y durante la lactancia (21 días con consumo de acuerdo a la demanda); T2 $(C Z n ; n=24)$ recibieron una dieta similar al T1, pero adicionada con $100 \mathrm{mg}$ de $Z \mathrm{n} / \mathrm{kg}$ de alimento, a partir de Metionina de Zinc (Met-Zn), durante el mismo periodo de tiempo y con el mismo manejo alimenticio.

El experimento se realizó durante los meses de enero a mayo de 2015 (época fresca); periodo durante el cual las cerdas estuvieron expuestas a un índice de temperatura y humedad (THI; Mader et al., 2006) entre normal (69 a 72; para los meses de enero a marzo), y alerta fisiológica (75 a 77; para los meses de abril y mayo). La temperatura ambiental promedio durante el periodo de estudio fue de $25.26^{\circ} \mathrm{C}$ y humedad relativa de $60.18 \%$.

\section{Experimento 2.}

Diseño experimental: se utilizaron 44 cerdas multíparas híbridas (Yorkshire $x$ Landrace), a las se les asignó uno de dos tratamientos en un diseño experimental completamente al azar. Los tratamientos $(T)$ consistieron en: $T 1(S Z n ; n=25)$; recibieron una dieta, a base de maíz-pasta de soya con aporte nutrimental de acuerdo a la etapa fisiológica, a partir de los 35 días de gestación (79 días, con un consumo de $2 \mathrm{~kg}$ alimento/día), y durante la lactancia (21 días con consumo a libre acceso); T2 (CZn; $n=19)$ recibieron una dieta similar al T1, pero adicionada con $100 \mathrm{mg}$ de Zn/kg de alimento, a partir de Met-Zn, durante el mismo periodo de tiempo y con el mismo manejo alimenticio.

El experimento se realizó durante los meses de junio a octubre de 2015, periodo durante el cual las cerdas estuvieron expuestas a un THI entre peligro ( 80 a 83, durante los meses de junio, agosto y octubre), y emergencia fisiológica (> 83) para los meses de julio y septiembre. La temperatura ambiental promedio durante el periodo de estudio fue de $31^{\circ} \mathrm{C}$ y humedad relativa de $68.24 \%$.

Manejo de los animales: las cerdas gestantes se alojaron en jaulas individuales (2.20 $\mathrm{m} \times 0.60 \mathrm{~m}$ ). Durante la gestación tuvieron libre acceso a agua de bebida y se proporcionaron $2 \mathrm{~kg} / \mathrm{d}$ de alimento de una dieta para cerdas gestantes (Cuadro 1), 
servidos durante la mañana $(07: 00 \mathrm{~h})$. Tres días antes de la fecha probable de parto fueron alojadas en jaulas individuales de maternidad $(2.20 \mathrm{~m} \times 1.50 \mathrm{~m})$, en salas cerradas con ventilación forzada; teniendo agua a libre acceso, y la alimentación se realizó tres veces al día de acuerdo a la demanda de consumo, con una dieta para hembras lactantes (Cuadro 1).

\begin{tabular}{|c|c|c|}
\hline Ingredientes & Gestación & Lactancia \\
\hline Maíz & 793 & 692 \\
\hline Pasta de soya & 160 & 254 \\
\hline Aceite & 5 & 18 \\
\hline Premezcla mineral & 42 & 36 \\
\hline \multicolumn{3}{|c|}{ Aporte nutrimental } \\
\hline E.M.(Mcal Kg $\left.{ }^{-1}\right)$ & 3.272 & 3.351 \\
\hline Proteína (\%) & 14.165 & 17.953 \\
\hline Lisina (\%) & 0.866 & 1.081 \\
\hline Fibra (\%) & 2.463 & 2.492 \\
\hline Fósforo (\%) & 0.596 & 0.699 \\
\hline Calcio (\%) & 0.980 & 0.915 \\
\hline
\end{tabular}

Cuadro 1. Composición e información nutricional de las dietas utilizadas en gestación y lactancia.

Mediciones: la medición del EGD se realizó a los 35 días de gestación (inicio de experimento), y tres días antes de la fecha probable de parto, a $65 \mathrm{~mm}$ a cada lado de la línea media, al nivel de la última costilla. Se registró el tamaño y peso de la camada al nacimiento, tamaño y peso de la camada al destete; así como el número de lechones muertos por camada durante la lactancia, y con base en ello se determinó el porcentaje de mortalidad durante este periodo. Además, se determinó la concentración de IgA en el calostro y la concentración plasmática de lgG e lgM en los lechones a los 14 d posdestete. Toma de muestras: las muestras para determinar la concentración de IgA fueron colectadas manualmente, después de haber nacido el primer lechón; se tomaron $2 \mathrm{ml}$ de calostro, el cual fue colocado en frascos estériles, identificados y mantenidos a temperatura ambiente durante 20 minutos; posteriormente se colocaron en hieleras a 4 ${ }^{\circ} \mathrm{C}$ para ser transportadas al laboratorio, donde fueron congeladas a $-20{ }^{\circ} \mathrm{C}$ hasta el momento de su análisis.

Las muestras de sangre para determinar la concentración plasmática de $\operatorname{lgG}$ e $\operatorname{lgM}$, fueron tomadas de los lechones a los 14 días posdestete de la vena yugular en tubos Vacutainer ${ }^{\circledR}$ para análisis de suero. La sangre se mantuvo a temperatura ambiente durante 20 minutos, para después colocarla en hieleras a $4{ }^{\circ} \mathrm{C}$ para ser transportadas al laboratorio, donde fueron centrifugadas a $3000 \mathrm{~g}$ durante 10 minutos para separar el suero, el cual fue congelado a $-20^{\circ} \mathrm{C}$ hasta el momento de su análisis.

Determinación de la concentración de inmunoglobulinas: la determinación del nivel de IgA en el calostro se realizó mediante una kit de ELISA (Pig IgA ELISA Kit Cat. No. 
E101-102 Lot No. E101-102-150306 de laboratorios Bethyl), y la determinación del nivel de IgG e IgM en suero de lechones a los 14 días posdestete, se realizó mediante un kit de ELISA (Pig IgG ELISA Kit Cat. No. E101-104 Lot No. E101-104-150206 y Pig IgM ELISA kit Cat. No. E101-117 Lot No. E101-117-150218 de laboratorios Bethyl).

Análisis estadístico: a los resultados de EGD, tamaño de camada al nacimiento, peso de la camada al nacimiento, peso de la camada al destete, tamaño de la camada al destete, concentración de $\lg A$ en calostro y concentración plasmática de $\lg G$ e $\lg M$ en cerdos 14 días posdestete, se les aplicó un análisis de varianza para un diseño completamente al azar (Steel y Torrie, 1985). Se fijó un alfa máximo de 0.05 para aceptar diferencia estadística y se consideró a cada cerda como la unidad experimental. El modelo matemático utilizado fue: $Y_{i j k}=\mu+Z n_{j}+E_{i j k}$; donde: $Y_{i}=$ variable de respuesta, $\mu$ $=$ media general del experimento, $Z n_{j}=E l$ efecto del $\mathrm{j}$ - ésimo nivel de Zinc y $E_{i j k}=$ Error aleatorio. A la tasa de mortalidad durante la lactancia se le aplicó un análisis de $X^{2}$, utilizando tablas de contingencia $2 \times 2$.

\section{RESULTADOS Y DISCUSIÓN}

Los resultados del experimento 1 (Época fresca), se muestran en los Cuadros 2, 3, y 4; los del experimento 2 (Época de calor), se resumen en los cuadros 5, 6 y 7. El consumo de alimento adicionado con $100 \mathrm{mg}$ de $\mathrm{Zn} / \mathrm{kg}$ a partir de Met-Zn no modificó $(P=0.28)$ el EGD de la cerda durante la época fresca del año (11.58 vs. $12.77 \mathrm{~mm}$ ); estos resultados coinciden con los obtenidos por Caine et al. (2009) quienes al proporcionar dietas adicionadas con $250 \mathrm{mg} / \mathrm{kg}$ de Zn orgánico (ZnAA), a partir del último tercio de gestación, no observaron modificaciones en esta variable; estos autores no reportaron las condiciones climáticas en las que se desarrolló el estudio.

En el presente estudio, la temperatura ambiental promedio durante el periodo en que se realizó el experimento 1 (Época fresca), fue de $25.26^{\circ} \mathrm{C}$ y humedad relativa de $60.18 \%$; condiciones ambientales que no provocan estrés por calor en las cerdas en gestación y lactancia (Mader et al., 2006). Sin embargo, el consumo adicional de Met-Zn, elevó ( $P=$ 0.05) el EGD (16.64 vs. $14.87 \mathrm{~mm}$ ) durante la época de calor (Exp. 2). La temperatura ambiental promedio durante este periodo de estudio fue de $31^{\circ} \mathrm{C}$ y humedad relativa de $68.24 \%$, condiciones ambientales que provocan estrés calórico en las cerdas gestantes y lactantes (Mader et al., 2006).

El estrés por calor ocurre cuando hay un desbalance entre la producción de calor y su disipación del cuerpo de los animales (Marai et al., 2007; Hansen, 2009; Bernabucci et al., 2010; Lewis y Bunter, 2011). La respuesta homeostática general al estrés calórico incluye una disminución en el consumo de alimento y un incremento en el consumo de agua, así como de la temperatura rectal, la temperatura de la piel y la tasa respiratoria en los cerdos (Pearce et al., 2013; Li et al., 2015). La disminución en el consumo de alimento es una respuesta adaptativa reconocida en muchas especies de animales (Baumgard y 
Rhoads, 2012), para disminuir la producción de calor metabólico (Renaudeau et al., 2012).

Otros investigadores han sugerido que el estrés calórico disminuye el crecimiento y la concentración plasmática de $\mathrm{Ca}, \mathrm{K}, \mathrm{Na}$, y Zn en los animales (Pearce et al., 2013); además de ocasionar una disminución en el consumo de nutrientes. El estrés calórico altera el metabolismo energético y la calidad de la canal en los cerdos, observándose que bajo ambientes con alta temperatura los animales ganan más tejido adiposo que lo energéticamente esperado (Pearce et al., 2013). Al respecto se ha informado que el Zn desempeña una importante función en el metabolismo de los lípidos en la célula, como parte funcional y estructural de algunas enzimas que intervienen en el metabolismo de los lípidos (Islam y Loots, 2007); y se ha sugerido que el consumo de dietas adicionadas con Zn incrementan la acumulación de grasa intramuscular en los lechones destetados a partir de la síntesis nueva de ácidos grasos libres, por una regulación en la expresión de genes trasportadores de ácidos grasos libres y por incrementar la actividad enzimática (Zhang et al., 2014). Qu et al. (2015), sugirieron la existencia de una respuesta celular autónoma al estrés calórico en los adipocitos de los cerdos, provocando una elevación de las reservas de lípidos en éstos, tal vez a través de la regulación positiva de los genes implicados en la absorción de ácidos grasos y la síntesis de triacilgliceridos.

Estos mismos autores, advirtieron que el estrés calórico aumenta la actividad de la lipoproteína lipasa (LPL) en el tejido adiposo de cerdos. Posteriormente Qu et al. (2016), informaron que el estrés calórico induce la expresión de la enzima fosfoenolpiruvato carboxiquinasa (PEPCK-C) en el tejido adiposo, provocando una elevación en la gliceroneogénesis, lo que podría explicar el aumento de la acumulación de grasa en los cerdos criados en ambiente con temperaturas elevadas.

La fosfoenolpiruvato carboxiquinasa (PEPCK) se encuentra en dos isoformas (PEPCKC y PEPCK-M); la PEPCKC se expresa principalmente en el hígado, el riñón y el tejido adiposo; en tanto que la PEPCK-M está presente en una variedad de tejidos no gluconeogénicos, incluyendo páncreas, cerebro, leucocitos, corazón o neuronas (Méndez-Lucas et al., 2014).

En un estudio previo Méndez-Lucas et al. (2013) concluyeron que la PEPCK-M tiene potencial gluconeogénico per se y coopera con la PEPCK-C para ajustar el flujo gluconeogénico a los cambios en la disponibilidad de sustrato o energía en el ciclo de Krebs, sugiriendo un papel en la regulación del metabolismo de glucosa y lípidos en el hígado. Méndez-Lucas et al. (2014) sugirieron que la PEPCK-M es importante para mantener la progresión y sobrevivencia celular bajo condiciones de estrés.

Los resultados obtenidos en el presente estudio, sugieren que el mayor EGD observado en las cerdas que consumieron alimento adicionado con Met-Zn durante la época de calor, pudo estar influenciado por el Zn, mejorando la actividad enzimática en la síntesis y deposición de tejido adiposo. 
El consumo de alimento adicionado con $100 \mathrm{mg}$ de zinc/kg, no modificó $(P>0.05)$ el número de lechones nacidos totales (LNT), lechones nacidos vivos (LNV), peso de la camada al nacimiento (PCN), lechones destetados (LD) y peso de la camada al destete (PCD); tanto en la época fresca del año como en la época de calor. Estos resultados coinciden con los obtenidos por otros investigadores en estudios previos. Al respecto, Vallet et al. (2014) no observaron mejoras en estas variables en cerdas alimentadas con una dieta que contenía $0.07 \%$ de sulfato de zinc desde los 80 días de gestación. Caine et al. (2009), al proporcionar una dieta adicionada con $250 \mathrm{mg} / \mathrm{kg}$ de un complejo de zincaminoácidos $(\mathrm{ZnAA})$ a las cerdas durante el último tercio de gestación, no observaron efecto $(p>0.10)$ en el crecimiento de los cerdos durante la lactancia.

El consumo de alimento adicionado con $100 \mathrm{mg}$ de $\mathrm{Zn} / \mathrm{kg}$ durante la época fresca (Exp. 1), no modificó $(P=0.70)$ el porcentaje de mortalidad de los lechones durante la lactancia (15.5 vs. 17\%); sin embargo, durante la época de calor (Exp. 2), el consumo adicional de Zn disminuyó $(P=0.006)$ el porcentaje de mortalidad durante la lactancia (11 vs. $26 \%)$. El zinc es un componente de muchas enzimas y por lo tanto contribuye en varias rutas fisiológicas, incluyendo la actividad antioxidante de la enzima $\mathrm{Cu} / \mathrm{Zn}$ superóxido dismutasa (Mistry y Williams, 2011), la actividad de las metaloproteasas (Balaban et al., 2012), en la transcripción (Swamynathan, 2010) y en la regulación de $\mathrm{CO}^{2}$ (anhidrasa carbónica; Sly y Hu, 1995). Cada uno de ellos podría contribuir a disminuir la mortalidad al nacimiento o antes del destete; sobre todo la regulación de $\mathrm{CO}^{2}$ por la anhidrasa carbónica.

Se ha sugerido que la asfixia durante el proceso de parto, contribuye tanto a la muerte fetal como a la mortalidad antes del destete (Alonso-Spilsbury et al., 2005). En relación a lo anterior, se ha informado que la mayor transferencia de zinc al feto se da durante el último tercio de la gestación (Mahan et al., 2009). Si es así, el zinc suplementario podría reducir la incidencia de muerte fetal durante el parto prolongado y la mortalidad antes del destete, aumentando la actividad de la anhidrasa carbónica, haciendo a los lechones más resistentes a altas concentraciones de $\mathrm{CO}^{2}$ durante el proceso de nacimiento, fundamentalmente en las cerdas sometidas a estrés calórico. Al respecto Ming-Zhe et al. (2016) informaron que el consumo de dietas adicionadas con $120 \mathrm{mg} / \mathrm{kg}$ de $\mathrm{Zn}$ a partir de Met-Zn, eleva la concentración sérica del Zn y la actividad de la anhidrasa carbónica en los lechones destetados. Caine et al. (2009) observaron que el consumo de dietas adicionadas con $250 \mathrm{mg} / \mathrm{kg}$ de $\mathrm{Zn}$ a partir de $\mathrm{ZnAA}$, durante el último tercio de gestación de las cerdas, eleva la concentración sérica de $Z n$ en los lechones lactantes; también indicaron que la administración gástrica por intubación de $40 \mathrm{mg} Z \mathrm{n}$ a partir de Met-Zn a los lechones lactantes al momento del nacimiento, a los 7 y $14 \mathrm{~d}$ de edad, mejoraba la condición de los lechones durante la lactancia. Vallet et al. (2014) obtuvieron una menor mortalidad predestete en cerdas alimentadas con una dieta adicionada con $0.07 \%$ de sulfato de zinc, desde los 80 días de gestación hasta el parto. 
Se ha sugerido que el $\mathrm{Zn}$ es requerido para apoyar la proliferación celular, y la diferenciación de tejidos en los órganos en desarrollo (Hyun-Ju et al., 2010; Zitka et al., 2010). Mahan et al. (2009) informaron que las cantidades de $\mathrm{Zn}, \mathrm{Cu}$ y Se que se transfieren al feto porcino, aumenta a medida que progresa la gestación; pero la mayor cantidad se transfiere durante los últimos 15 días de gestación. Estos micro minerales se incorporan en varias enzimas y sistemas enzimáticos antioxidantes del cuerpo; por lo tanto, su mayor concentración en los fetos durante la gestación puede reflejar un mayor potencial de actividad antioxidante durante esta etapa. Esto es importante de considerar en líneas de cerdas de alta producción criadas bajo condiciones de estrés calórico, ya que, si la dieta de la cerda no contiene los niveles dietéticos adecuados de minerales para satisfacer las necesidades reproductivas, la cerda movilizará las reservas corporales antes del inicio de la lactancia, lo que puede comprometer la lactancia de la cerda y el rendimiento de su camada.

Los lechones provenientes de cerdas que consumieron alimento adicionado con $\mathrm{Zn}$ durante la época fresca, tuvieron una mayor $(P=0.001)$ concentración plasmática de lgG (267 vs. $390.8 \mathrm{ng} / \mathrm{mL}$ ) a los 14 días posdestete (Cuadro 4); en tanto que la concentración de IgM no fue modificada (214.1 vs. $207.1 \mathrm{ng} / \mathrm{mL}$ ), al igual que la concentración de lgA en el calostro (1057 vs. $1001 \mathrm{ng} / \mathrm{mL}$ ). Durante la época de calor los niveles plasmáticos de $\operatorname{lgG}(373.3$ vs. $310 \mathrm{ng} / \mathrm{mL}$ ) e $\operatorname{lgM}(235.1 \mathrm{vs} .221 .8 \mathrm{ng} / \mathrm{mL})$ no fueron modificados por el consumo de dietas adicionadas con Met-Zn; sin embargo, en las cerdas que no recibieron $Z n$ adicional, se observó una tendencia $(P=0.068)$ de incremento en la concentración de IgA en el calostro (1100.6 vs. $1017.9 \mathrm{ng} / \mathrm{mL}$ ).

Estudios previos sugieren que las concentraciones de $\lg A$ e $\lg G$ en el calostro están influenciadas por la estación del año. Se ha observado que exponer a las cerdas a estrés por frío durante los últimos 10 días antes del parto, puede aumentar la absorción de lgG por los lechones (Bate y Hacker, 1985); sin embargo, cuando los lechones son sometidos a estrés por frío, reducen las concentraciones plasmáticas de lgG, presumiblemente por la reducción en la ingesta de calostro (Blecha y Kelley, 1981). También se ha informado que los valores de IgG disminuyen en verano y otoño, o cuando las cerdas son expuestas a altas temperaturas al final de la gestación (Machado-Neto et al., 1987). Inoue (1981) informó que los valores de IgA disminuyen en primavera, verano y otoño, pero aumentan en invierno. Los niveles más bajos de IgA en el calostro de las cerdas que consumieron alimento adicionado con Met-Zn, se pudo deber al efecto farmacológico del Zn sobre la flora patógena intestinal, disminuyendo o estabilizando su población en el intestino; así como al mantenimiento de la integridad intestinal, lo que evita que ésta sea permeable a las endotoxinas bacterianas. Se ha informado al respecto que la suplementación con ZnO se ha asociado con una disminución en el traslado de bacterias desde el intestino delgado a los nódulos linfáticos mesentérico (Huang et al., 1999) y con un incremento en la 
estabilidad y homogeneidad en la población de coliformes (Kautouli et al., 1999); así como protegiendo la integridad de la mucosa intestinal (Pearce et al., 2015).

Además de la barrera física que proporcionan los epitelios, el sistema inmunológico de la mucosa también utiliza otros tejidos linfoides asociados con el intestino (TLAI), para proteger al organismo y mediar respuestas innatas y adaptativas subsiguientes. Una característica distintiva de la inmunidad de la mucosa intestinal es la inducción de una respuesta inmune en las placas de Peyer y la producción subsiguiente de IgA, por linfocitos B en la lámina propia (Burkey et al., 2009), cuando es estimulada por un agente antigénico.

Los mayores niveles plasmáticos de lgG (267 vs. $390.8 \mathrm{ng} / \mathrm{mL}$ ) observados en los lechones provenientes de cerdas que recibieron dietas adicionadas con Met-Zn durante la época fresca, se pudo deber al mayor consumo de esta inmunoglobulina a través del calostro y al efecto conocido del Zn de mejorar la respuesta inmune. Se sabe que el Zn es esencial para las células de alta proliferación, especialmente en el sistema inmunológico, e influye tanto en las funciones inmunes innatas como en las adquiridas (Maggini et al., 2007; Haase y Rink, 2009b; Mocchegiani et al., 2009). Deficiencias de Zn disminuyen la función de las células $T$ y baja los títulos de anticuerpos (Richards et al., 2010). Se ha informado que la adición de $\mathrm{Zn}$ a partir de metionina de zinc, mejora la repuesta inmune celular y humoral en gallinas (Soni et al., 2013).

En un estudio reciente Ming-Zhe et al. (2016) informaron que tanto la suplementación con $120 \mathrm{mg} / \mathrm{kg}$ de $\mathrm{Zn}$ a partir de Met-Zn o de nano partículas de ZnO, eleva los niveles plasmáticos de lgG en lechones destetados.

\begin{tabular}{|c|c|c|c|c|}
\hline \multirow[t]{2}{*}{ Variable } & \multicolumn{2}{|l|}{ Tratamientos } & \multirow[t]{2}{*}{ EEM $^{1}$} & \multirow[t]{2}{*}{ Valor de $\mathrm{P}$} \\
\hline & Testigo & Met-Zn² & & \\
\hline Observaciones $(\mathrm{n})$ & 22 & 24 & & \\
\hline$E \mathrm{GD}^{3} 35$ d de gestación $(\mathrm{mm})$ & 8.95 & 9.08 & 0.3537 & 0.86 \\
\hline EGD 111 d de gestación $(\mathrm{mm})$ & 12.77 & 11.58 & 0.5473 & 0.28 \\
\hline $\mathrm{LNT}^{4}$ & 13.68 & 13.21 & 0.4617 & 0.61 \\
\hline LNV $^{5}$ & 12.18 & 11.63 & 0.4777 & 0.57 \\
\hline $\mathrm{PCN}^{6}(\mathrm{~kg})$ & 16.19 & 16.20 & 0.5561 & 0.99 \\
\hline $\mathrm{LD}^{7}$ & 10.09 & 9.83 & 0.2130 & 0.55 \\
\hline $\mathrm{PCD}^{8}$, ajustada a $21 \mathrm{~d}$ de lactancia $(\mathrm{kg})$ & 53.352 & 54.802 & 1.4671 & 0.66 \\
\hline
\end{tabular}

Cuadro 2. Efecto del consumo de alimento adicionado con 100 ppm de Zinc orgánico en el desempeño productivo de cerdas gestantes y lactantes durante la época fresca del año. 


\begin{tabular}{|c|c|c|c|}
\hline \multirow[t]{2}{*}{ Variable } & \multicolumn{2}{|l|}{ Tratamientos } & \multirow[t]{2}{*}{ Valor de P } \\
\hline & Testigo & Met-Zn ${ }^{1}$ & \\
\hline Observaciones (n) & 22 & 24 & \\
\hline LNV $^{2}$ & 12.18 & 11.63 & \\
\hline $\mathrm{LD}^{3}$ & 10.09 & 9.83 & \\
\hline Mortalidad durante la lactancia (\%) & 17 & 15.5 & 0.70 \\
\hline
\end{tabular}

${ }^{1}$ Metionina de zinc, ${ }^{2}$ Lechones nacidos vivos, ${ }^{3}$ Lechones destetados

Cuadro 3. Efecto del consumo de alimento adicionado con 100 ppm de Zinc orgánico en la época fresca del año en la tasa de mortalidad durante la lactancia.

\begin{tabular}{|c|c|c|c|c|}
\hline \multirow[t]{2}{*}{ Variable } & \multicolumn{2}{|c|}{ Tratamiento } & \multirow[t]{2}{*}{ EEM $^{1}$} & \multirow[t]{2}{*}{ Valor de $\mathrm{P}$} \\
\hline & Testigo & Met-Zn² & & \\
\hline Hembras, $n$ & 22 & 24 & & \\
\hline $\operatorname{lgA}, \mathrm{ng} / \mathrm{mL}$ & 1057.2 & 1001.4 & 35.9 & 0.448 \\
\hline Lechones, n & 20 & 20 & & \\
\hline $\mathrm{lgG}, \mathrm{ng} / \mathrm{mL}$ & 267 & 390.8 & 19.8 & 0.001 \\
\hline $\mathrm{lgM}, \mathrm{ng} / \mathrm{mL}$ & 214.1 & 207.1 & 11.5 & 0.765 \\
\hline
\end{tabular}

1 Error estándar de la media, ${ }^{2}$ Metionina de zinc

Cuadro 4. Influencia del consumo de alimento adicionado con $\mathrm{Zn}$ durante la época fresca en los niveles de IgA en el calostro y niveles plasmáticos de IgM e IgG en los lechones 14 días posdestete.

\begin{tabular}{|c|c|c|c|c|}
\hline \multirow[t]{2}{*}{ Variable } & \multicolumn{2}{|l|}{ Tratamientos } & \multirow[t]{2}{*}{$\mathrm{EEM}^{1}$} & \multirow[t]{2}{*}{ Valor de $\mathrm{P}$} \\
\hline & Testigo & Met-Zn² & & \\
\hline Observaciones (n) & 25 & 19 & & \\
\hline EGD 35 d de gestación (mm) & 14.64 & 14.55 & 0.4999 & 0.93 \\
\hline EGD 111 d de gestación (mm) & 14.87 & 16.64 & 0.5903 & 0.05 \\
\hline $\mathrm{LNT}^{4}$ & 12.64 & 11.55 & 0.4334 & 0.21 \\
\hline LNV $^{5}$ & 10.44 & 9.35 & 0.4660 & 0.24 \\
\hline $\mathrm{PCN}^{6}(\mathrm{~kg})$ & 12.55 & 12.34 & 0.5768 & 0.85 \\
\hline $\mathrm{LD}^{7}$ & 7.72 & 8.31 & 0.2971 & 0.32 \\
\hline $\mathrm{PCD}^{8}$, ajustada a $21 \mathrm{~d}$ de lactancia $(\mathrm{kg})$ & 42.28 & 45.58 & 1.8582 & 0.38 \\
\hline
\end{tabular}

1 Error estándar de la media, ${ }^{2}$ Metionina de zinc, ${ }^{3}$ Espesor de grasa dorsal, ${ }^{4}$ Lechones nacidos totales, ${ }^{5}$ Lechones nacidos vivos, ${ }^{6}$ Peso de la camada al nacimiento, ${ }^{7}$ Lechones destetados $y^{8}$ Peso de la camada al destete

Cuadro 5. Efecto del consumo de alimento adicionado con 100 ppm de Zinc orgánico en el desempeño de cerdas gestantes y lactantes durante la época cálida del año.

\begin{tabular}{lccc}
\hline Variable & Tratamientos & \multirow{2}{*}{ Valor de P } \\
\cline { 2 - 3 } & Testigo & Met-Zn $^{1}$ \\
\hline Observaciones $(\mathrm{n})$ & 25 & 19 & 9.35 \\
LNV $^{2}$ & 10.44 & 8.31 & \\
LD $^{3}$ & 7.72 & 11 & 0.006 \\
Mortalidad durante la lactancia (\%) & 26 & \\
\hline
\end{tabular}

${ }^{1}$ Metionina de zinc, ${ }^{2}$ Lechones nacidos vivos, ${ }^{3}$ Lechones destetados

Cuadro 6. Efecto del consumo de alimento adicionado con 100 ppm de Zinc orgánico durante la época cálida del año en la tasa de mortalidad durante la lactancia. 


\begin{tabular}{|c|c|c|c|c|}
\hline \multirow[t]{2}{*}{ Variable } & \multicolumn{2}{|c|}{ Tratamiento } & \multirow[t]{2}{*}{ EEM $^{1}$} & \multirow[t]{2}{*}{ Valor de $\mathrm{P}$} \\
\hline & Testigo & Met-Zn² & & \\
\hline Hembras, $n$ & 25 & 19 & & \\
\hline $\lg A, n g / m L$ & 1100.6 & 1017.9 & 22.7 & 0.068 \\
\hline Lechones, $n$ & 24 & 23 & & \\
\hline $\operatorname{lgG}, \mathrm{ng} / \mathrm{mL}$ & 373.3 & 310 & 29.5 & 0.288 \\
\hline $\mathrm{lgM}, \mathrm{ng} / \mathrm{mL}$ & 235.1 & 221.8 & 16.5 & 0.691 \\
\hline
\end{tabular}

1 Error estándar de la media, ${ }^{2}$ Metionina de zinc

Cuadro 7. Influencia del consumo de alimento adicionado con metionina de Zn durante la época de calor, en los niveles de IgA en el calostro y niveles plasmáticos de IgM e IgG en los lechones 14 días posdestete.

\section{CONCLUSIÓN}

El consumo de dietas adicionadas con $100 \mathrm{mg}$ de $\mathrm{Zn}$ por $\mathrm{kg}$ de alimento, aumenta el espesor de grasa dorsal y disminuye el porcentaje de lechones muertos durante la lactancia, en cerdas bajo estrés calórico; el consumo de alimento adicionado con $\mathrm{Zn}$ durante la época fresca incrementa los niveles de IgG en los lechones con 14 días posdestete.

\section{LITERATURA CITADA}

ALONSO-SPILSBURY MD, Mota-Rojas, Villanueva-García D, Martínez-Burnes J, Orozco H, Ramírez-Necoechea R, Mayagoitia AL, Trujillo ME. 2005. Perinatal asphyxia pathophysiology in pig and human: A review. Animal Reproduction Science. 90(1-2):130. ISSN: 0378-4320, http://dx.doi.org/10.1016/..anireprosci.2005.01.007

BALABAN NP, Rudakova NL, Sharipova MR. 2012. Structural and functional characteristics and properties of metzincins. Biochemistry (Moscow). ISSN: 1608-3040, 77(2):119-127. http://dx.doi.org/10.1134/S0006297912020010

BAO YM, M. Choct, lji PA, Bruerton K. 2007. Effect of organically complexed copper, iron, manganese, and zinc on broiler performance, mineral excretion, and accumulation in tissues. The Journal of Applied Poultry Research. 16:448-455. ISSN: 1537-0437, https://doi.org/10.1093/japr/16.3.448

BATE LA, Hacker RR. 1985. The influence of the sow's adrenal activity on the ability of the piglet to absorb IgG from colostrum. Canadian Journal of Animal Science. 65(1):7785. ISSN: 0008-3984, http://dx.doi.org/10.4141/cjas85-008

BAUMGARD LH, Rhoads RP. 2012. Ruminant production and metabolic responses to heat stress. Journal of Animal Science. 90(6):1855-1865. ISSN: 1525- 3163, http://dx.doi.org/10.2527/jas.2011-4675.

BERNABUCCI U, Lacetera N, Baumgard LH, Rhoads RP, Ronchi B, Nardone A. 2010. Metabolic and hormonal acclimation to heat stress in domesticated ruminants. Animal. 4(7):1167-1183. ISSN: 1751-732X, https://dx.doi.org/10.1017/S175173111000090X 
BHOWMIK D, Chiranjib KP, Sampath K. 2010. A potential medicinal importance of zinc in human health and chronic disease. International Journal Pharmaceutical and Biomedical Research. 1(1):05-11.

ISSN:

2348-0262 https://www.researchgate.net/publication/277014212 A potential medicinal importance of zinc in human health and chronic disease

BLECHA F, Kelley KW. 1981. Cold stress reduces the acquisition of colostral immunoglobulin in piglets. Journal of Animal Science. 52:594-600. ISSN: 1525- 3163, http://agris.fao.org/agris-search/search.do?recordID=US19820749155

BORAH S, Sarmah BC, Chakravarty P, Naskar S, Dutta DJ, Kalita D. 2014. Effect of zinc supplementation on serum biochemicals in grower pig. Journal of Applied Animal $\begin{array}{llllll}\text { Research. } & 42 & (2): & 244- & 248 . & \text { ISSN: }\end{array}$ http://dx.doi.org/10.1080/09712119.2013.824888

BURKEY TE, Skjolaas KA, Minton JE. 2009. BOARD-INVITED REVIEW: Porcine mucosal immunity of the gastrointestinal tract. Journal of Animal Science. 87(4):14931501. ISSN: 1525- 3163, http://dx.doi.org/10.2527/jas.2008-1330

CAINE WR, Metzler-Zebeli BU, McFall M, Miller B, Ward TL, Kirkwood RN, Mosenthin R. 2009. Supplementation of diets for gestating sows with zinc amino acid complex and gastric intubation of suckling pigs with zinc-methionine on mineral status, intestinal morphology and bacterial translocation in lipopolysaccharide-challenged early-weaned pigs. Research in Veterinary Science. 86(3):453-462. ISSN: 0034-5288, http://dx.doi.org/10.1016/j.rvsc.2008.10.005. Epub 2008 Dec 4

CHAND N, Naz S, Khan A, Khan S, Khan RU. 2014. Performance traits and immune response of broiler chicks treated with zinc and ascorbic acid supplementation during cyclic heat stress. International Journal of Biometeorology. 58 (10):2153-2157. ISSN: 1432-1254 http://dx.doi.org/10.1007/s00484-014-0815-7

CHASAPIS CT, Loutsidou AC., Spiliopoulou CA, Stefanidou ME. 2012. Zinc and human health: an update. Archives of Toxicology. 86:521-534. ISSN: 0340-5761, http://dx.doi.org/10.1007/s00204-011-0775-1

HAASE H, Rink L. 2009a. The immune system and the impact of zinc during aging. Immunity and Ageing. 6:9. ISSN: 1742-4933, http://dx.doi.org/10.1186/1742-4933-6-9

HAASE H, Rink L. 2009b. Functional significance of zinc-related signalling pathways in immune cells. Annual Review of Nutrition. 29:133-152. ISSN: 1545-4312, http://dx.doi.org/10.1146/annurev-nutr-080508-141119

HANSEN PJ. 2009. Effects of heat stress on mammalian reproduction. Philosophical Transactions of the Royal Society of London B. 364(1534):3341-3350. ISSN: 0080-4614, https://dx.doi.org/10.1098/rstb.2009.0131 
HILL GM, Mahan DC, Jolliff JS. 2014. Comparison of organic and inorganic zinc sources to maximize growth and meet the zinc needs of the nursery pig. Journal of Animal Science. 92:1582-1594. ISSN: 1525- 3163, http://dx.doi.org/10.2527/jas2013-6744

HU CH, Song ZH, Xiao K, Song J, Jiao LF, Ke YL. 2014. Zinc oxide influences intestinal integrity, the expressions of genes associated with inflammation and TLR4-myeloid differentiation factor 88 signaling pathways in weanling pigs. Innate Immunity. 20:478486. ISSN: 17534267, http://dx.doi.org/10.1177/1753425913499947

HUANG SX, McFall M, Cegielski AC, Kirkwood RN. 1999. Effect of dietary zinc supplementation on Escherichia coli septicemia in weaned pigs. Journal of Swine Health and Production. 7:109-111. ISSN: 1537-209X https://www.aasv.org/shap/issues/v7n3/v7n3p109.pdf

HYUN-JU S, Young-Eun C, Taewan K, Hong-In S, In-Sook K. 2010. Zinc may increase bone formation through stimulating cell proliferation, alkaline phosphatase activity and collagen synthesis in osteoblastic MC3T3-E1 cells. Nutrition Research and Practice. 4(5):356-361. ISSN:

2005-6168, www.ncbi.nlm.nih.gov/pmc/articles/PMC2981717/pdf/nrp-4-356.pdf

INOUE T. 1981. Possible factors influencing immunoglobulin: A concentration in swine colostrum. American Journal of Veterinary Research. 42:533-536. ISSN: 0002-9645, http://europepmc.org/abstract/med/7271021

ISLAM M, Loots DT. 2007. Diabetes, metallothionein and zinc interations: a review. Biofactors. 29 (4):203-212. ISSN: 1872-8081, http://dx.doi.org/10.1002/biof.5520290404

KAUTOULI M, Melin L, Jensen-Waern M, Wallgren P, Mollby R. 1999. The effect of zinc oxide supplementation on the stability of the intestinal flora with special reference to composition of coliformes in weaned pigs. Journal of Applied Microbiology. 87:564-573. ISSN: 1365-2672, http://dx.doi.org/10.1046/j.1365-2672.1999.00853.x

KELLEHER S, McCormick NH, Velasquez V, Lopez V. 2011. Zinc in specialized secretory tissues: Roles in the pancreas, prostate, and mammary gland. Advances in Nutrition. 2:101-111. ISSN: 2156-5376, http://advances.nutrition.org/content/2/2/101.full.pdf+html

LAGANA C, Ribeiro AML, Kessler A, Kratz LR, Pinheiro CC. 2007. Effect of the supplementation of vitamins and organic minerals on the performance of broilers under heat stress. Revista Brasileira de Ciencia Avícola. 9(1):01-06. ISSN: 1806-9061, http://dx.doi.org/10.1590/S1516-635X2007000100006

LEWIS CRG, Bunter KL. 2011. Effects of seasonality and ambient temperature on genetic parameters for production and reproductive traits in pigs. Animal Production Science. 51:615-626. ISSN, 1836-0939, http://dx.doi.org/10.1071/AN10265 
LI Y, Cao Y, Zhou X, Wang F, Shan T, Li Z, Xu W, Li C. 2015. Effects of zinc sulfate pretreatment on heat tolerance of Bama miniature pig under high ambient temperature. Journal of Animal Science. 93:3421-3430. ISSN: 1525- 3163, http://dx.doi.org/10.2527/jas.2015-8910

MACHADO-NETO R, Graves CN, Curtis SE. 1987. Immunoglobulins in piglets from sows heat-stressed prepartum. Journal of Animal Science. 65(2):445-455. ISSN: 1525- 3163, http://dx.doi.org/10.2527/jas1987.652445x

MADER TL, Davis MS, Brown-Brandl T. 2006. Environmental factors influencing heat stress in feedlot cattle. Journal of Animal Science. 84:712-719. ISSN: 1525- 3163, http://digitalcommons.unl.edu/cgi/viewcontent.cgi?article $=1622 \&$ context=animalscifacpu $\underline{\mathrm{b}}$

MAGGINI S, Wintergerst ES, Beveridge S, Hornig DH. 2007. Selected vitamins and trace elements support immune function by strengthening epithelial barriers and cellular and humoral immune responses. The British Journal of Nutrition. 1:29-35. ISSN: 1475-2662, http://dx.doi.org/10.1017/S0007114507832971

MAHAN DC, Watts MR, St-Pierre N. 2009. Macro- and micromineral composition of fetal pigs and their accretion rates during fetal development. Journal of Animal Science. 87:2823-2832. ISSN: 1525- 3163, http://www.prairieswine.com/pdf/40184.pdf

MARAI IFM, El-Darawany AA, Fadiel A, Abdel-Hafez MAM. 2007. Physiological traits as affected by heat stress in sheep: a review. Small Ruminant Research. 71:1-12. ISSN: 0921-4488, http://doi.org/10.1016/j.smallrumres.2006.10.003

MÉNDEZ-LUCAS A, Duarte JA, Sunny NE, Satapi S, He TT, Fu X, Bermúdez J, Burguess SC, Perales JC. 2013. PEPCK-M expression in mouse liver potentiates, not replaces, PEPCK-C mediated gluconeogenesis. Journal of Hepatology. 59 (1): 105-113. ISSN: 0168-8278, http://dx.doi.org/10.1016/i.jhep.2013.02.020

MÉNDEZ-LUCAS A, Hyroššová P, Novellasdemunt L, Viñals F, Perales JC. 2014. Mitochondrial phosphoenolpyruvate carboxykinase (PEPCK-M) is a pro-survival, endoplasmic reticulum (ER) stress response gene involved in tumor cell adaptation to nutrient availability. The Journal of Biological Chemistry. 289 (32): 22090-102. ISSN . 0021-9258, http://dx.doi.org/10.1074/jbc.M114.566927

MING-ZHE L, Jie-Ting H, Yi-Hao T, Syuan-Yian M, Chao-Ming F, Tu-Fa L. 2016. Nanosize of zinc oxide and the effects on zinc digestibility, growth performances, immune response and serum parameters of weanling piglets. Animal Science Journal. 87: 13791385. ISSN: 1740-0929, http://dx.doi.org/10.1111/asj.12579 
MISTRY HD, Williams PJ. 2011. The importance of antioxidant micronutrients in pregnancy. Oxidative Medicine and Cellular Longevity. ID 841749, 12 pages. ISSN: $1942-$ 0994, http://dx.doi.org/10.1155/2011/841749

MOCCHEGIANI E, Giacconi R, Cipriano C, Malavolta M. 2009. NK and NKT cells in aging and longevity: role of zinc and metallothioneins. Journal of Clinical Immunology. 29:416425. ISSN: 1573-2592, http://dx.doi.org/10.1007/s10875-009-9298-4

NOLLET L, Van der Klis JD, Lensing M, Spring P. 2007. The Effect of replacing inorganic with organic trace minerals in broiler diets on productive performance and mineral excretion. The Journal of Applied Poultry Research. 16:592-597. ISSN: 1537-0437, https://doi.org/10.3382/japr.2006-00115

NRC (National Research Council). 2012. Nutrient requirements of swine. 11th rev. ed. Natl. Acad. Press, Washington, DC. ISBN: 978-0-309-22423-9

PAYNE RL, Bidner TD, Fakler TM, Southern LL. 2006. Growth and intestinal morphology of pigs from sows fed two zinc sources during gestation and lactation. Journal of Animal Science. 84:2141-214. ISSN: 1525- 3163, http://dx.doi.org/10.2527/jas.2005-627

PEARCE SC, Gabler NK, Ross JW, Escobar J, Patience JF, Rhoads RP, Baumgard LH. 2013. The effects of heat stress and plane of nutrition on metabolism in growing pigs. Journal of Animal Science. 91:2108-2118. ISSN: 1525- 3163, http://dx.doi.org/10.2527/jas.2012-5738

PEARCE SC, Sanz FMV, Torrison J, Wilson ME, Baumgard LH, Gabler NK. 2015. Dietary organic zinc attenuates heat stress-induced changes in pig intestinal integrity and metabolism. Journal of Animal Science. 93:4702-4713. ISSN: 1525- 3163, http://dx.doi.org/10.2527/jas2015-9018

QU H, Donkin SS, Ajuwon KM. 2015. Heat stress enhances adipogenic differentiation of subcutaneous fat depot-derived porcine stromovascular cells. Journal of Animal Science. 93(8):3832-3842. ISSN: 1525- 3163, http://dx.doi.org/10.2527/jas.2015-9074

QU H, Yan H, Lu H, Donkin SS, Ajuwon KM. 2016. Heat stress in pigs is accompanied by adipose tissue-specific responses that favor increased triglyceride storage1. Journal of Animal Science. 94(5):1884-1896. ISSN: 1525- 3163, http://dx.doi.org/10.2527/jas2015$\underline{0084}$

RENAUDEAU D, Collin A, Yahav S, de Basilio V, Gourdine JL, R. Collier J. 2012. Adaptation to tropical climate and research strategies to alleviate heat stress in livestock production: A review. Animal. 6(5):707-728. ISSN: 1751-732X, http://dx.doi.org/10.1017/S1751731111002448 
RICHARDS JD, Zhao J, Harrell RJ, Atwell CA, Dibner JJ. 2010. Trace Mineral Nutrition in Poultry and Swine. Asian-Australian Journal of Animal Science. 23 (11):1527-1534. ISSN, 1011-2367, http://www.ajas.info/upload/pdf/23-200.pdf

RICHARDS JD, Fisher PM, Evans JL, Wedekind KJ. 2015. Greater bioavailability of chelated compared with inorganic zinc in broiler chicks in the presence or absence of elevated calcium and phosphorus. Open Access Animal Physiology. 7:97-109. ISSN: 1179-2779, https://doi.org/10.2147/OAAP.S83845

SANZ FMV, Pearce SC, Gabler NK, Patience JF. 2014. Effects of supplemental zinc amino acid complex on gut integrity in heat-stressed growing pigs. Animal. 8:43-50. ISSN: 1751-732X, http://dx.doi.org/10.1017/S1751731113001961

SCHLEGEL P, Sauvant D, Jondreville C. 2013. Bioavailabiliy of zinc sources and their interaction with phytates in broilers and piglets. Animal. 7(1):47-59. ISSN: 1751-732X, http://dx.doi.org/10.1017/S1751731112001000

SLY WS, Hu PY. 1995. Human carbonic anhydrases and carbonic anhydrase deficiencies. Annual Review of Biochemistry. 64:375- 401. ISSN: 1545-4509, http://dx.doi.org/10.1146/annurev.bi.64.070195.002111

SONG ZH, Ke YL, Xiao K, Jiao LF, Hong QH, Hu CH. 2015. Diosmectite-zinc oxide composite improves intestinal barrier restoration and modulates TGF- $\beta 1$, ERK1/2, and Akt in piglets after acetic acid challenge. Journal of Animal Science. 93:1599-1607. ISSN: 1525- 3163, http://dx.doi.org/10.2527/jas.2014-8580

SONI NEETA, Mishra SK, Swain R, Das A, Chichilichi B, Sethy K. 2013. Bioavailability and Immunity Response in Broiler Breeders on Organically Complexed Zinc Supplementatio. Food and Nutrition Sciences. 4: 1293-1300. ISSN: 2157-9458, http://dx.doi.org/10.4236/fns.2013.412166

STEEL GD, Torrie JH. 1985. Bioestadística: Principios y Procedimientos. (2da. Ed.) McGraw-Hill, México, D. F. ISBN: 0-07-060926-8

SWAMYNATHAN S. 2010. Krüppel-like factors: Three fingers in control. Humans Genomics. 4(4):263-270. ISSN: 1479-7364, http://dx.doi.org/10.1186/1479-7364-4-4-263

VALLET JL, Rempel LA, Miles JR, Webel SK. 2014. Effect of essential fatty acid and zinc supplementation during pregnancy on birth intervals, neonatal piglet brain myelination, stillbirth, and preweaning mortality. Journal of Animal Science. 92(6):2422-2432. ISSN: 1525- 3163, https://dx.doi.org/10.2527/jas.2013-7130

ZHANG B, Guo Y. 2009. Supplemental zinc reduced intestinal permeability by enhancing occludin and zonula occludens protein-1 (ZO-1) expression in weaning piglets. The British 
Journal of Nutrition. 102:687-693. ISSN: 0007-1145, http://dx.doi.org/10.1017/S0007114509289033

ZHANG HB, Wang MS, Wang ZS, Zhou AM, Zhang XM, Dong XW, Peng QH. 2014. Supplementation dietary zinc levels on growth performance, carcass traits, and intramuscular fat deposition in weaned piglet. Biological Trace Element Research. 161:6977. ISSN: 1559-0720, http://dx.doi.org/10.1007/s12011-014-0078-5

ZITKA O, Kukacka J, Krizkova S, Huska D, Adam V, Masarik M, Prusa R, Kizek R. 2010. Matrix Metalloproteinases. Current Medicinal Chemistry. 17:3751-3768. ISSN: 09298673,

http://web2.mendelu.cz/af 239 nanotech/data/pub/Matrix\%20Metalloproteinases.pdf 